\title{
The Introduction of OpenOffice.org in the Brussels Public Administration
}

\author{
Kris Ven, Dieter Van Nuffel, and Jan Verelst \\ University of Antwerp, Department of Management Information Systems, \\ Faculty of Applied Economics \\ Prinstraat 13, B-2000 Antwerp, Belgium \\ \{kris.ven, dieter.vannuffel, jan.verelst\}Qua.ac.be
}

\begin{abstract}
Open source software is increasingly used by public administrations as an alternative to commercial software. In this paper we present a case study of the transition of the ministerial cabinets of the Brussels-Capital Region towards OpenOffice.org. In this case, the decision to use open source software was taken by the Government of the Brussels-Capital Region. The goal of the paper is to outline the implementation trajectory followed and to compare our findings to previous studies in this field as well as other Information Systems literature. Additionally, we discuss how OpenOffice.org was received by end users as well as the IT department that was responsible for the migration. Our findings indicate that although a migration towards OpenOffice.org is feasible, a number of difficulties still remain. For example, end user perceptions of OpenOffice.org are not always favorable and migration costs (document conversion and training) can be significant.
\end{abstract}

Key words: open source, adoption, public administration, OpenOffice.org

\section{Introduction}

Open standards and open source software are increasingly used by public administrations (PA) in Europe. A recent survey in 13 European countries has shown that $49 \%$ of PAs intentionally use open source software, while another $29 \%$ make unaware use of open source software. Moreover, half of the respondents would find an increase in open source software usage useful [1]. This increased use of open source software has received the attention of a number of European research projects, such as the Open Source Observatory ${ }^{1}$ of the IDABC (Interoperable Delivery of European eGovernment Services to public Administrations, Businesses and Citizens) and more recently the COSPA (Consortium for Open Source in the Public Administration) project ${ }^{2}$.

Although open source software is mainly used on servers, it can be argued that the use of open source software on the desktop could result in considerable

${ }^{1}$ http://europa.eu.int/idabc/en/chapter/452

2 http: //www. cospa-project.org

Please use the following format when citing this chapter:

Ven, K., Van Nuffel, D., and Verelst, J., 2006, in IFIP International Federation for Information Processing, Volume 203, Open Source Systems, eds. Damiani, E., Fitzgerald, B., Scacchi, W., Scotto, M., Succi, G., (Boston: Springer), pp. 123-134 
higher savings in license costs; since the number of desktop licenses is much larger than that of server licenses. On the other hand, migrating towards open source software on the desktop is far more disruptive for end users and will result in higher migration costs than a migration on server level (e.g. when migrating from MS Internet Information Services to Apache). These factors could explain why the use of open source desktop software is quite limited. Nevertheless, about $20 \%$ of PAs included in the FLOSSPOLS study indicated that they make use of OpenOffice.org [1].

Hence, we feel that additional research is required to assess if and how migrations towards open source software could take place. In this paper, we will report on the transition to open source software by the Brussels-Capital Region. The transition primarily concerned the use of OpenOffice.org by the ministerial cabinets of the Government of the Brussels-Capital Region. The rest of the paper is structured as follows. We will start in Sect. 2 by describing the background of the transition. In Sect. 3, the methodology of our present research is discussed. Section 4 describes the implementation trajectory that was followed during the transition. In Sect. 5, experiences from end users and the IT department responsible for the implementation are presented. Finally, conclusions are drawn for future migrations.

\section{Background}

According to the revision of the Belgian Constitution in 1970, Belgium was officially divided into three regions: the Flemish Region, the Walloon Region and the Brussels-Capital Region. The Brussels-Capital Region consists of the 19 communes of Brussels and is administered by two authorities: the Parliament and the Government of the Brussels-Capital Region. The latter consists of eight Ministries, each having its own cabinet. The Brussels-Capital Region has two official languages: Dutch and French. This requires for example that official documents are composed in these two languages. In 1987, a law was passed that created the Brussels Regional Informatics Center (BRIC). The responsibilities of BRIC include the promotion and assistance of Information Technology (IT) in the local public administrations of the Brussels-Capital Region.

The move towards open standards and open source software by the BrusselsCapital Region was initiated by two decisions. First, a resolution was voted in which the use of open standards and open source software was encouraged in the Brussels-Capital Region in order to facilitate the communication with the citizens of the region. As a result, BRIC was required to consider at least one open source alternative in each project. Second, based on this resolution, the use of open standards and open source software was included in the coalition agreement of the Brussels-Capital Region in 2004. It was decided by the Government of the Brussels-Capital Region that open source office software would be used for the ministerial cabinets of the Brussels-Capital Region. OpenOffice.org was however not mentioned by name. 
Within BRIC, OpenOffice.org was introduced in April 2004. In a second phase, from mid-January until mid-February 2005, the ministerial cabinets were migrated to OpenOffice.org. The transition concerned a total of 400 workstations running Windows XP on which OpenOffice.org 1.1 was installed. Apart from the desktops, 4 out of 8 servers of the cabinets were migrated from MS Windows to Linux.

\section{Methodology}

In order to describe the introduction of OpenOffice.org in the Brussels Public Administration, we opted for a descriptive case study approach. This approach enabled us to describe the phenomenon in its real-life context $[2,3]$. The case study used an embedded design, since the use of OpenOffice.org was investigated at BRIC as well as the ministerial cabinets of the Brussels-Capital Region.

Since BRIC was responsible for the IT services both within BRIC and within the ministerial cabinets, we selected two respondents within BRIC using the key informant method, namely the director of the IT department and the project leader who was assigned to the OpenOffice.org project. This allowed us to gather more information, since it has been shown that the use of a single respondent leads to unreliable results $[3,4]$. Both respondents where closely involved in the migration towards OpenOffice.org. They were responsible for tasks such as planning and coordinating the migration, developing documentation, designing the training sessions and conducting user evaluations.

A first interview took place to gather important background information on the case study. Based on this information, a case study protocol was crafted, including a detailed set of questions, the data collection procedures and the outline of the case study report. The primary mode of data collection was a face-to-face interview which was recorded for future reference. This interview was conducted by a two person team: one researcher was responsible for posing the interview questions, while the other was responsible for taking notes and supplement the interview with additional questions. This also allowed for viewing the case from two perspectives and compare the impressions of both researchers afterwards $[2,5]$. Additional sources of evidence were internal documents of BRIC, legislative texts and secondary information such as press releases. Follow-up questions were asked via e-mail. A draft copy of the case study report was reviewed by the respondents in our interview to increase the validity of our findings. The findings of this case study were further compared to findings of previous studies on the adoption of OpenOffice.org and other Information Systems (IS) literature to further ground our conclusions.

\section{Implementation}

In this section, we will discuss the different phases in which the implementation of OpenOffice.org took place. We will discuss successively the analysis, the 
training offered to end users, the actual migration and the conversion of document templates. We will finish by discussing the current developments of the project.

\subsection{Analysis}

The IDA Open Source Migration Guidelines [6] prescribe making a detailed business case for a possible migration towards open source software. The business case should include making a Total Cost of Ownership (TCO) analysis of the various alternatives (proprietary vs. open source solutions) over a certain time period. Migration costs should be included in this analysis.

Although no formal TCO analysis was performed in this case, the main driver of the adoption of OpenOffice.org was cost reduction. The reduction in license costs amounts to a total of 185,000 euro in the first year and 15,000 euro in the following years (a number of remaining workstations will be migrated in the following years, resulting in additional savings). Some authors have warned against a focus on cost savings alone during the adoption of open source software [7].

On the other hand, in compliance with the IDA recommendations, a pilot project was initiated at BRIC in March 2004 to study the feasibility of a transition from MS Office to OpenOffice.org. The result of the pilot project confirmed the feasibility of migrating the ministerial cabinets to OpenOffice.org.

\subsection{Training}

The importance of training when starting to use a new software package has been described in previous case studies on OpenOffice.org, as well as in traditional IS literature $[8,9,10,11]$. Training increases the proficiency of end users with the software which in turn increases user acceptance.

The training of end users included a training course in the offices of BRIC and a CD-ROM with additional information. The training course consisted of a voluntary one-day session in which the basic functionality of both OpenOffice.org Writer and Calc was explained. For the first sessions, key users of each cabinet (the cabinet clerk and an IT responsible) were invited in order to be able to offer first-line support for users in their cabinet. Two out of eight ministerial cabinets considered the training to be too basic. Since participation in the training was voluntary, these two cabinets decided not to encourage staff members of their cabinet to attend the training sessions. It could be observed in a survey among end users (see Sect. 4.5) that users who reported not having attended the training session also reported more problems in using OpenOffice.org.

As some authors note $[7,12]$, users should be able to start practice with OpenOffice.org immediately after training, in order to maximize the effectiveness of the training session. Given the narrow time frame of one month that was allocated to the migration (see Sect. 4.3 for the exact time frame), this was 
impossible to achieve. However, all staff workers received their training within one week before or after their workstation was migrated to OpenOffice.org.

At the end of the training session, a CD-ROM containing a manual, a FAQ list and the installation files of OpenOffice.org was handed out to the participants. The manual and FAQ were mainly adopted from the OpenOffice.org communities on the Internet. It should be noted however that significant differences between the OpenOffice.org localization communities can be established. While the French community is very active and provides much documentation, the Dutch counterpart does not achieve the same quality. A possible explanation could be that the number of native French speakers is much higher than the number of native Dutch speakers. However, this caused no significant problems since the majority of staff members at the ministerial cabinets was native French speaking, and the majority of Dutch speaking staff members experienced little problems in studying the French material.

\subsection{Migration}

As previously noted, the migration was mandatory for staff members of the ministerial cabinets following the coalition agreement of 2004. Computer equipment of the ministerial cabinets is updated every five years, coinciding with the terms of the Government of the Brussels-Capital Region. For reasons of efficiency and to minimize discomfort for end users, it was decided to migrate to OpenOffice.org 1.1 at the same time the user's workstation was replaced. Hence, the phasing out of MS Office and the installation of OpenOffice.org were performed simultaneously. This means that MS Office was no longer available when OpenOffice.org was installed on the user's 'workstation, except in a limited number of cases in which the user required advanced functionality of MS Excel or MS Access (e.g. in the finance department). Concurrently, the default data format for internal communication changed from MS Office to OpenOffice.org format. When corresponding with external parties, the export filters of OpenOffice.org were used to save the document in MS Office format. Thanks to these import/export, filters, it was decided not to convert existing MS Office documents to the OpenOffice.org format.

Some authors have cautioned against this "big bang" approach since this would increase user resistance towards adopting the software $[6,7,12]$. In this case, it was judged that it was more convenient to immediately switch to OpenOffice.org, without temporarily installing MS Office. It was further expected that users with an initial negative attitude towards OpenOffice.org would continue using MS Office until it was deleted from their workstation. Additionally, changing the default document format to OpenOffice.org format further increased the social norm for staff members to use OpenOffice.org. On the other hand, it will be interesting to analyze the feedback of staff members (see Sect. 4.5) with respect to the migration to check whether this strategy did have an impact on the acceptance of OpenOffice.org. 


\subsection{Document Templates}

A problem that occurred during the migration to OpenOffice.org concerned the use of document templates. The Brussels PA has issued a very rigorous style guide to which all documents within the PA must adhere. BRIC tried to migrate the existing MS Office templates into an equivalent OpenOffice.org template. However several differences between MS Office and OpenOffice.org exist which led to incompatibilities, like for instance incorrect margins. Currently, BRIC is finalizing the OpenOffice.org templates that comply with the style guide of the Brussels PA.

A related difficulty consisted of editing legislative texts because of the very specific format that has to be used. Since Brussels is bilingual, the text must be published in two columns (one for each language) and each paragraph must start on the same level as the corresponding paragraph in the other language (see Fig. 1). Since French paragraphs are in general somewhat lengthier than the Dutch, some adjustments in vertical spacing between paragraphs must be made. In MS Word, a table with two columns and one row was used to realize this layout. However, OpenOffice.org does not support table cells to be spread over more than one page. Therefore, end users had to change the layout and store each paragraph in a different cell. When a cell (i.e. paragraph) does not fit on the bottom of the page, the cell is moved to the following page.

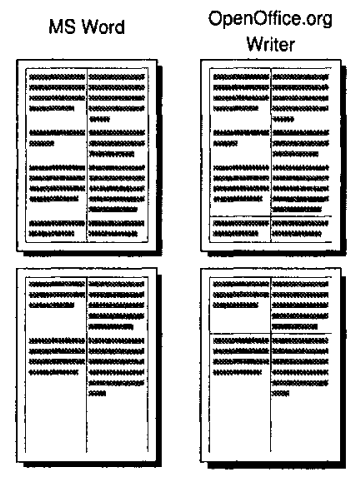

Fig. 1. Formatting of legislative texts in MS Office vs. OpenOffice.org

\subsection{Current Developments}

With the aim of enhancing end user support in future projects, BRIC recently carried out a survey within the ministerial cabinets in order to gather feedback on the migration. In the survey, staff members are asked about their use of 
OpenOffice.org, the training that was received and the experiences with OpenOffice.org so far. Although analysis of the results is still being performed, we can already report on preliminary experiences of end users in Sect. 5 .

Following the availability of OpenOffice.org 2.0, BRIC internally migrated to this new version in the beginning of December 2005. The OpenDocument format is currently used as the default data format for communication within BRIC, while the OpenOffice.org 1.1 format is still used when communicating with the ministerial cabinets. This upgrade was also performed to prepare a possible migration of the ministerial cabinets towards this new version. Partly based on these experiences, the ministerial cabinets have decided at the end of December 2005 to upgrade to OpenOffice.org 2.0.

\section{Experiences}

In this section, we will report on the experiences of end users of the ministerial cabinets and BRIC. We will focus on a number of issues that were encountered during the migration, and which lessons were drawn from these experiences.

\subsection{User Experiences}

As reported in previous studies, the initial attitude of end users towards using OpenOffice.org is an important factor in the success of the transition $[7,12,13]$. While technical problems can generally be solved, user resistance is much harder to overcome. In this case, it can indeed be observed that users who were not convinced before or at the time of training are still opposed to using OpenOffice.org. This confirms the importance of training and information dissemination towards users before the migration takes place.

Traditional literature on individual adoption also emphasized the importance of perceived ease of use and perceived usefulness of a technology in order to increase end user acceptance $[14,15,16]$. With respect to usability, the perception of staff members at the ministerial cabinets is mixed. In general, users find the usability of OpenOffice.org 1.1 to be lacking compared to MS Office. The most often heard critique is that the look and feel of OpenOffice.org feels outdated. A second difficulty for end users is that some tasks in OpenOffice.org should be performed slightly differently than in MS Office. It was observed that many users tried to work in the same manner they were used to in MS Office, which sometimes caused problems, for example when working with formatting styles. In those cases, BRIC used a procedural training approach [17] in which the preferred procedure was shown during a short personal demonstration. This was sufficient in most cases to alter the end users' old habits.

Another critique of some end users is that OpenOffice.org still lacks functionality that is present in MS Office. These users do not consider OpenOffice.org a fully fledged alternative for MS Office. More analysis of the survey results needs to be performed to establish whether the features reported missing by users are 
important in their daily tasks, since a previous study on the adoption of OpenOffice.org by PAs in Italy [7, 12] has shown that the features of OpenOffice.org are more than adequate for daily use. This mixed perception by end users is consistent with the observations from a previous experiment in a PA [8]. It is further hoped that the upgrade from OpenOffice.org version 1.1 to version 2.0 will improve its perception.

It has also been suggested in literature that users may resist working with open source desktop software, because they fear becoming deskilled by moving away from the industry standard $[6,18,19]$. According to our respondents at BRIC, this fear was not articulated by end users. In addition, while some authors note that the switch from proprietary systems to open source software may result in a decreased productivity shortly after the introduction [8, 19], no noticeable differences were observed. It must however be noted that no formal measurement of the productivity was performed.

\subsection{Evaluation}

The transition to OpenOffice.org has resulted in a number of benefits, but also involved a number of problems. A first advantage is that OpenOffice.org uses open standards to save documents [20]. As mentioned in Sect. 2, the support of open standards was an important factor in the decision towards using OpenOffice.org. On the other hand, despite the fact that the OpenOffice.org format is an open standard, it is yet only supported by OpenOffice.org. Hence, when communicating with external parties, documents must be converted to the MS Office format. Although the conversion performed by OpenOffice.org works adequately in most cases, some problems may arise when MS Office documents are poorly formatted, or when documents are converted multiple times back and forth between MS Office and OpenOffice.org format. These issues are also reported in previous studies [13]. Within the ministerial cabinets, most documents requiring input from multiple parties mainly involve revisions in the document text. Therefore, it was agreed upon that documents would be exchanged without formatting between parties, and only when the document was finalized, formatting was applied either in MS Office or OpenOffice.org. The use of the Portable Document Format (PDF) was also promoted for documents requiring no further modifications.

A second advantage of OpenOffice.org was its use of data sources to allow users to communicate with external databases. Especially the fact that a document can access more than one data source is very valuable. On the other hand, the implementation of these data sources in OpenOffice.org caused some difficulties at first. The reference to the data source is not included in the document as it is done by MS Office, but is stored in the user profile at the user's workstation. Therefore, when the document is exchanged between users, the data source is lost. This required custom scripting to ensure that each user has access to the data sources. Another related problem that was reported by end users was the Mail Merge feature. When using the Mail Merge feature to create 
a mailing based on an address list in a database, OpenOffice.org creates a new document for each addressee. Therefore, a script was developed to merge these documents back into one file. In OpenOffice.org 2.0 however, this script has become obsolete, since the user is given the choice between generating a separate file for each addressee, or to generate one large file.

As mentioned in Sect. 4.5, BRIC is already using OpenOffice.org version 2.0 since December 2005. In their experience, it appears that most of the difficulties mentioned above are solved in this new version. In general, OpenOffice.org 2.0 is considered to be an important improvement compared to version 1.1. Apart from the improved functionality, the look and feel more closely resembles that of MS Office which will make it easier to use than the previous version. The key users of each ministerial cabinet have already been migrated to OpenOffice.org 2.0 to provide feedback on this new version. First impressions of these key users confirm that OpenOffice.org 2.0 indeed solves a number of issues that were present in OpenOffice.org 1.1. On the other hand, it is noted by BRIC that OpenOffice.org 2.0 still contains a number of important defects. Although workarounds are possible for most of these problems, they could still have a negative impact on the general perception of OpenOffice.org.

Finally, several authors have noted that migration costs for training, document conversion and building up expertise can be an important barrier to the adoption of open source software $[19,21,22]$. In the past 18 months, BRIC needed to invest a lot of time in building up expertise in OpenOffice.org, developing training material and rewriting several templates that are in use by the ministerial cabinets. However, as mentioned in Sect. 4.1, a TCO analysis was not performed, neither before nor after the implementation. Hence, it is not possible at this time to assess whether the migration has resulted in a lower TCO. Nevertheless, these migration costs only occur once and could be compensated by additional savings in license costs in the following years. Moreover, the time that BRIC has invested in OpenOffice.org has paid off. Thanks to the experience with the prior installation of OpenOffice.org, BRIC was able to reduce the time required for installation considerably. While the initial installation of OpenOffice.org 1.1 at BRIC required 4 days, the upgrade to version 2.0 was completed in 1 day.

\section{Conclusion}

In this paper we reported on the migration of BRIC and the ministerial cabinets of the Government of the Brussels-Capital Region to OpenOffice.org. Our study has a number of contributions. First, we provided insight into how the migration to OpenOffice.org was undertaken by BRIC. Second, the main contribution of this paper is that it further builds upon previous studies on the adoption of OpenOffice.org as well as traditional IS literature. Hence, the findings of our case study were contrasted with the findings of previous studies. This allowed us to further validate these findings and provided the necessary grounding of 
our findings in literature. Third, we discussed which successes and shortcomings were encountered during the transition towards OpenOffice.org. It was established that while it is possible to migrate from MS Office to OpenOffice.org for most daily use of an office productivity suite, a number of issues required special attention which are of interest for potential adopters of OpenOffice.org.

We have seen that there were a number of technical issues that arose during the migration towards OpenOffice.org. Much effort was spent on the conversion of organization-specific templates. Additionally, some of the issues we discussed were specific to the environment under study, and were for example due to the bilingualism of Brussels. As a result of these issues, users had to alter their way of working when formatting a document. The import and export filters of OpenOffice.org were deemed sufficient in most cases, although some incompatibilities arose when repeatedly saving a file back and forth in OpenOffice.org and MS Office format. A work-around for this problem has however been devised. User feedback also indicated that OpenOffice.org was not considered a fully fledged alternative for MS Office by most users. For example, some advanced features of MS Office were not supported, and the look and feel of OpenOffice.org was considered outdated by end users. It is however expected by BRIC that the upgrade to OpenOffice.org 2.0 could alleviate a number of important nuisances which will improve its perception by end users. This is supported by the feedback received from the key users who have already been migrated to OpenOffice.org 2.0. This new version however still contains a number of issues which may negatively influence the end user experience.

The migration performed by BRIC also confirmed the importance of proper training for end users. Although the training that was provided by BRIC concerned basic office tasks, it was noted that users who attended the training session reported less problems in their daily tasks. Furthermore, the training sessions also proved to be important in creating an initial positive attitude towards OpenOffice.org, since it was observed that attitudes of end users are difficult to alter after the implementation. Although training material and documentation was available from the OpenOffice.org community, it was observed that there were considerable differences in quantity and quality between the Dutch and French community material. Hence, potential adopters should take differences between language communities into account when using documentation produced by the OpenOffice.org community.

While most of our findings are consistent with previous studies on the migration towards OpenOffice.org, we have found a number of contradictions with previous studies. Based upon the information we obtained from our respondents, users within BRIC and the ministerial cabinets did not articulate a fear for becoming deskilled or did not suffer from any noticeable lapses in productivity shortly after the upgrade. Additionally, the "big bang" approach that was taken did not seem to have a negative impact on the user's perceptions. However, detailed analysis of the user evaluations is required in order to further verify these results. 
Thanks to the migration towards OpenOffice.org, BRIC was able to realize a significant reduction in license costs. It remains however unclear whether the resulting TCO of OpenOffice.org in this case was indeed lower, since there were considerable migration costs including training, conversion of templates and building up expertise with OpenOffice.org. The result of these efforts will however be useful in the following years, so these initial costs could be compensated by additional savings in license costs in the future. Organizations and public administrations that are willing to adopt OpenOffice.org should however be aware that the transition will require significant investments in training and data migration, yet the long-term effects of the transition should also be taken into account.

\section{References}

1. Ghosh R, Glott R (2005) Results and policy paper from survey of government authorities. Technical report, MERIT, University of Maastricht, Maastricht. Free/Libre and Open Source Software: Policy Support (FLOSSPOLS) Deliverable D3

2. Yin RK (2003) Case study research: Design and methods. Sage Publications, Newbury Park, California, 3rd edition

3. Benbasat I, Goldstein DK, Mead M (1987) The case research strategy in studies of information systems. MIS Quarterly 11(3):368-386

4. Phillips LW (1981) Assessing measurement error in key informant reports: A methodological note on organizational analysis in marketing. Journal of Marketing Research (JMR) 18(4):395-415

5. Eisenhardt KM (1989) Building theories from case study research. Academy of Management Review 14(4):532-550

6. European Communities (2003) The IDA open source migration guidelines. http: //europa.eu.int/idabc/servlets/Doc?id=1983

7. Zuliani P, Succi G (2004a) Migrating public administrations to open source software. In: Proceedings of e-Society 2004 IADIS International Conference. Avila, Spain

8. Rossi B, Scotto M, Sillitti A, Succi G (2005) Criteria for the non invasive transition to openoffice. In: Scotto M, Succi G. (eds) Proceedings of the First International Conference on Open Source Systems. Genova, Italy, pp. 250-253

9. Kovács GL, Drozdik S, Zuliani P, Succi G (2004a) Open source software for the public administration. In: Proceedings of the 6th Computer Science and Information Technologies (CSIT). Budapest, Hungary

10. Nelson RR, Cheney PH (1987) Training end users: An exploratory study. MIS Quarterly 11(4):546-559

11. Thompson RL, Higgins CA (1991) Personal computing: Toward a conceptual model of utilization. MIS Quarterly 15(1):125-143

12. Zuliani P, Succi G (2004b) An experience of transition to open source software in local authorities. In: Proceedings of e-Challenges on Software Engineering. Vienna, Austria

13. Drozdik S, Kovács GL, Kochis PZ (2005) Risk assessment of an open source migration project. In: Scotto M, Succi G (eds) Proceedings of the First International Conference on Open Source Systems. Genova, Italy, pp. 246-249 
14. Davis FD (1989) Perceived usefulness, perceived ease of use, and user acceptance of information technology. MIS Quarterly $13(3): 319-340$

15. Venkatesh V, Davis FD (2000) A theoretical extension of the technology acceptance model: Four longitudinal field studies. Management Science 46(2):186-204

16. Venkatesh V, Morris MG, Davis GB, Davis FD (2003) User acceptance of information technology: Toward a unified view. MIS Quarterly 27(3):425-478

17. Olfman L, Mandviwalla M (1994) Conceptual versus procedural software training for graphical user interfaces: A longitudinal field experiment. MIS Quarterly 18(4):405-426

18. Fitzgerald B, Kenny $\mathrm{T}$ (2003) Open source software in the trenches: Lessons from a large scale implementation. In: Proceedings of 24th International Conference on Information Systems (ICIS). Seattle, Washington

19. Fitzgerald B (2005) Has open source software a future? In: Feller J, Fitzgerald B, Hissam S, Lakhani K (eds) Perspectives on free and open source software. MIT Press, Cambridge, MA, pp. 93-106

20. Kovács GL, Drozdik S, Zuliani P, Succi G (2004b) Open source software and open data standards in public administration. In: Proceedings of the IEEE International Conference on Computational Cybernetics. Vienna, Austria

21. Goode S (2005) Something for nothing: Management rejection of open source software in Australia's top firms. Information \& Management 42(5):669-681

22. Dedrick J, West J (2004) An exploratory study into open source platform adoption. In: Proceedings of the 37th Hawaii International Conference on System Sciences. IEEE Computer Society, Washington, DC 